\title{
Structure and algorithms of motion control system's software of the small spacecraft
}

\author{
Filatov A.V., \\ Progress Space Rocket Centre, Samara \\ Tkachenko I.S., Tyugashev A.A., Sopchenko E.V. \\ Samara State Aerospace University
}

\begin{abstract}
The paper is devoted to some features and algorithms of AIST-2D experimental technological small spacecraft's Motion Control System Software. Special Flight Control Software is embedded in Motion Control System. There are several modes of software functioning depending on state of the spacecraft. There are descriptions of the basic software algorithms in the paper.

Keywords: Motion Control System, Flight Control Software, Real-Time Control Algorithm, Program Turn

Citation: Filatov A.V.,Tkachenko I.S., Tyugashev A.A., Sopchenko E.V. Structure and algorithms of motion control system's software of the small spacecraft. Proceedings of Information Technology and Nanotechnology (ITNT-2015), CEUR Workshop Proceedings, 2015; 1490: 246-251. DOI: 10.18287/1613-0073-2015-1490-246-251
\end{abstract}

\section{Introduction}

Software is a very important part of every modern space project. The errors and glitches of Flight Control Software could lead to critical consequences including losses of expensive spacecrafts. Onboard computational opportunities are the basis for successful execution of widespread spectrum of tasks in long space flights [1]. This issue is important even for micro- and nano- satellites which represent the very fast growing segment of the spacecrafts.

The Motion Control System (MCS) of the AIST-2D small spacecraft includes both hardware (sensors, devices and aggregates) and software [2].

The MCS should perform the following:

- quieting of the spacecraft after separation from the launch vehicle;

- orientation of the spacecraft with a given accuracy in the Orbital Coordinate System;

- orientation of the solar panels' surfaces towards the Sun with a given accuracy;

- self-diagnostic information's forming and transmission to Earth; 
- form and send information about the spacecraft angular position to the Onboard Control System (OCS) upon request;

- support the mode without control pulses.

After the separation from the launch vehicle's upper stage or after the Undirected Flight Mode (UFM), the Mode of Compensation of the Initial Angular Velocity is being started by activation of Executive and Sensing Elements Complex (ESEC). When the angular velocity reaches the given value, Single-Axis Solar Orientation Mode will start automatically. This mode supported until the moment of request from the Onboard Control System to start Three-Axis Orientation in Orbital Coordinate System Mode. The switching between the MCS modes is possible by transmission of the special command messages. Timing Diagram of the MCS functioning is being presented in Fig. 1.

So, in accordance with its purpose, MCS supports the following set of modes:

- Compensation of the Initial Angular Velocity Mode (MCIAV);

- Single-Axis Solar Orientation Mode (MSASO);

- Three-Axis Orientation in Orbital Coordinate System Mode (MTAORS);

- MCS experiments Mode (MMET).

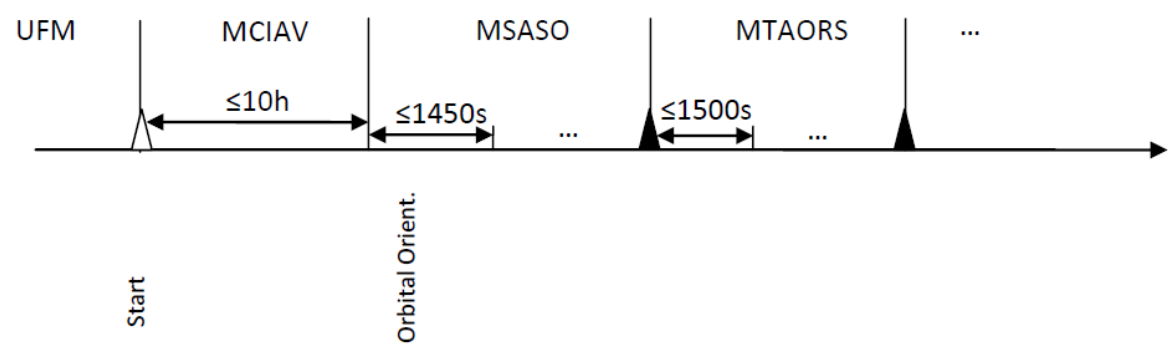

Fig. 1. - Motion Control System Functioning Timing Diagram

\section{Modes Description}

Compensation of the Initial Angular Velocity Mode starts after its activation and should compensate the angular velocity of the spacecraft. The mentioned angular velocity could be formed after the separation from the upper stage or during the Undirected Flight.

With decreasing kinetic moment of the apparatus to a predetermined value (corresponding to the angular velocity of 0.07 degrees per second), the MCS automatically activates (without control actions from the OCS) stabilization using the Reaction Wheels.

Next, on the time interval of $100 \mathrm{sec}$, the process of angular velocity damping process is being performed with the use of Reaction Wheels.

MCIAV ends automatically by the transition to Single-Axis Solar Orientation Mode. 
Single-Axis Solar Orientation Mode designed to support orientation the surfaces of the solar panels towards the Sun with a given accuracy.

The Mode starts automatically after the end of the MCIAV or by the request from the OCS. The command message from OCS is being transmitted using the special channel of information exchange.

The first operations of the MSASO are activation of the Orbital Solar Sensors (OSS) and checking of the Sun's detection. If the Sun detection process completed successfully by at least the one OSS, the Program Turn into Solar Orientation will be activated.

If the Sun cannot be detected, the spacecraft begins the rotation to find the Sun.

The maximal duration of the Sun Search process depends on angular velocity and guaranteed review of the full sphere by the Sensor's Visual Field. Thus, the maximal duration of the search will not exceed $600 \mathrm{sec}$ (excluding time of shadow).

The velocity of the search is 1 degree per second.

After the Program Turn, the stabilization in Solar Orientation begins. In this case, the combination of the axis of OYSCS with the direction vector to the Sun in Associated Coordinate System should be carried out.

The Mode continues until reception of command message from the OCS to change the MCS mode.

Three-Axis Orientation in Orbital Coordinate System Mode should support Spacecraft's orientation in the Orbital Coordinate System with the given accuracy and support the Program of Angle Motion Control. This Program implements the Time Control Method and starts upon request from OCS.

The Mode starts by the command message from OCS via information exchange channel.

The first actions of the Mode are the following. The Search of Earth (SOE) process starts and it checks if the Earth detected in the spacecraft's field of view. If there is no Earth in the field of view in initial position, the SOE should be executed by the spacecraft's rotation.

The maximal duration of the SOE process depends on angular velocity and guaranteed review of the full sphere by the Sensor's Visual Field. The maximal duration of the search is not exceeds $300 \mathrm{sec}$. The velocity of the search is 1 degree per second.

After the successful end of Earth's detection process, the pre-defined sequence of algorithm's segments will be implemented. After completion of the mentioned segments, the spacecraft will have the right orientation in Orbital Coordinate System and will be ready for implementation of Program of Angle Motion Control.

This Program can be initiated by the one of the following:

1. Array containing the time of turn's start and the quaternion defines the position of the Solar Coordinate System in the Orbital Coordinate System at the moment of the end of turn;

2. Array of the parameters $\left(\mathrm{A}_{\mathrm{i}}, \omega_{\mathrm{i}}, \varphi_{\mathrm{i}}\right)$ which define angle position of Solar Coordinate System relatively to Orbital Coordinate System, by the three channels. In this case, Program of Angle Motion Control is being formed by MCS in accordance with the 
expression: $\psi_{\mathrm{i}}=\mathrm{A}_{\mathrm{i}} \cdot \sin \left(\omega_{\mathrm{i}} \cdot \mathrm{t}+\varphi_{\mathrm{i}}\right)$, considering correspondingly the angles of roll, yaw, pitch.

3. Array containing the polynomials of approximation - Program of Angle Motion Control for the each channel.

The Mode executes until receiving the command message from the OCS to change the mode of functioning.

MCS experiments Mode should support tests of the MCS equipment and checking of the experimental algorithms of the MCS functioning.

The Mode starts by the command message from the OCS to implement the tests or experiments. Which devices of MCS will be used, depends on the program of experiment.

The Mode is active until the command message from the OCS to change the mode of functioning.

To effective implementation of the all described modes of MCS functioning, the development of the AIST-2D software includes the following steps:

- Development of the technical overviews of the software algorithms;

- Programming;

- Unit testing;

- Integration testing using the emulator of Earth's Debug Testbed Complex;

- Complex testing and debugging of the software using the Earth's Debug Testbed Complex.

All algorithms of the MCS's onboard software can be divided into the following categories:

System Algorithms:

- Planning of the MCS functioning;

- Collection of telemetry information and its output to OCS;

- Forming of control information related to angle motion of the spacecraft;

Algorithms of the MCS Modes:

- Solar System Coordinates Orientation of the spacecraft;

- Orbital System Coordinates Orientation of the spacecraft;

- MCS control in case of technical faults;

- MCS tests and experiments;

Device control algorithms:

- Fiber-optic gyroscopes control;

- Reaction Wheels control;

- Star trackers control;

- Magnetic Executives control;

- Magnetometers control ;

- Optical Solar Sensors control;

- Earth orientation devices control; 
Algorithms of Stabilization:

- Spacecraft Motion Control by Reaction Wheels;

- Kinetic Moment Clear;

- Program Turn;

- Calculation of Control Parameters;

- Program of Angle Motion Control Forming;

- Algorithms of Orientation Detection;

- Solar Orientation;

Algorithms of Control:

- Control of Redundancy in case of technical faults;

- Control of Stabilization using Reaction Wheels;

- Control of Magnetic System;

Algorithms of Experiments:

- Orientation Detection using Angle Velocity measurement and data from Star Trackers;

- Orientation Detection in Inertial Coordinate System using Star Trackers.

\section{Conclusion}

Thus, algorithms and Flight Control software of AIST-2D small spacecraft's MCS provide:

1. Compensation of the angle velocity after the separation from the upper stage of the launch vehicle (duration of the compensation process not more than 3 hours when angle velocity less than 7 degrees per second and 2 hours 15 minutes when angle velocity less than 3 degrees per second);

2. Orientation in Orbital Coordinate System with the error less than (with probability $\mathrm{P}=0,96$ ):

(a) by the angle position

(i) not worse than 30 angle minutes (without additional navigation information);

(ii) not worse than 30 angle minutes in of pitch and roll channels и 10 angle minutes in yaw channel (with usage navigation information);

(b) by angle velocity - 0,005 degrees per second;

3. One-axis solar panels orientation towards the Sun with the error not exceeds $(\mathrm{P}=0,96)$ :

(a) in angle position -3 degrees;

(b) in angle velocity - 0,5 degrees per seconds in all channels;

4. Maximal angle velocities of the spacecraft during Program Turn -1 degree per second;

5. Maximal angle acceleration during Program Turn - 0,029 degrees per quadrate seconds;

6. Transition from Solar Orientation into Orbital Orientation and in reverse order: 
(a) 10 minutes (using additional data about center of mass position);

(b) 25 minutes (without navigation information).

7. Reaching of maximal accuracy in Orbital Coordinate System for no more than 35 minutes from the moment of building of Orbital Orientation.

\section{Acknowledgment}

The authors would like to thank the reviewers for the valuable comments and suggestions. Development of the Earth observation small spacecraft, including development of the algorithms and software, is being carried out in accordance with the Complex Project of Samara Progress Rocket Center and Samara Space Aerospace University «Creation of the high-technology manufacture of small Earth observation spacecrafts with the using of hyper-spectral devices for the social and economical development of Russia and international cooperation» (this work is partially supported by the Government of the Russia in accordance with Decree No. 218 dated 09.04.2010). This work was also supported by the Ministry of Education and Science of the Russian Federation.

\section{References}

1. Journal Aircraft Engineering. Vol. 6: The results of science and technology VINITI. Moscow, 1998. [in Russian]

2. Sevastyanov NN, Branets VN, Panchenko VA, Kazinskiy NV, Kondranin TV, Negodyaev SS. The analysis of the modern opportunities of small Earth observation satellites' development. Proceedings of MIPT, 2009; 1(3). [in Russian] 